\title{
Enhancing efficacy in laser projection by four wavelength combination
}

\author{
Ian Wallhead, Roberto Ocaña* and Paula Quinzá \\ AIDO, Instituto Tecnológico de Óptica, C/Nicolás Copérnico, \\ 7-13, Parque Tecnológico, 46980 Paterna, Valencia, Spain \\ *Corresponding author: rocana@aido.es, robertoocagna@gmail.com
}

\begin{abstract}
In this letter we present a novel study about the combination of four laser wavelengths in order to maximize both color gamut and efficacy to produce the color white. Firstly, an analytic method to calculate efficacy as function of both four laser wavelengths and four laser powers is derived. Secondly we provide a new way to present the results by providing the diagram efficacy vs color gamut area that summarizes the performance of any wavelength combination for projection purposes. The results indicate that the maximal efficacy for the D65 white is only achievable by using a suitable combination of both laser power ratios and wavelengths.
\end{abstract}

PACS numbers:

Mobile projection devices (usually known as picoprojectors) should be designed to maximize their luminous efficacy. This is for two main reasons. Firstly, being either stand alone devices or embedded in other products, they are likely to be powered by battery, and lifetime an important factor [1]. Secondly, the increasing use of lasers to project images calls for a consideration of eye safety issues [2]. The brightness of the projected image may be limited by the Class II accessible emission limit. There is reason to believe that current laser beam scanning picoprojector technology is already close to the power ceiling based on eye safety limits [3]. Consequently, it would be desirable to improve luminous efficacy to increase the output luminous flux for the same eye-safe optical power limit.

The relationship between the choice of laser wavelengths and the resulting luminous efficacy has been discussed in the literature [4 $[$ ] . Here we present the opportunity to increase luminous efficacy by adding a fourth laser wavelength. Usually, the three primary wavelengths of a laser display offer a wide color gamut and so the emphasis in this study has been to maximize the safe luminous flux whilst maintaining the gamut, rather than increasing the gamut. Utilization of additional wavelengths or colors in display and illumination applications is however not new. Reasons for adding one or more additional colors have traditionally been for improved gamut and/or improved brightness and have usually applied to liquid crystal display applications. Brown et al. 7] have discussed adding a white pixel to the red, green and blue pixels of an LCD display to increase the brightness of a display. Sharp has recently launched a four-color television with claims of increased brightness and color gamut [8].

Here we present a study of the choices of four laser wavelengths to optimize luminous efficacy for the projection of the CIE standard illuminant D65 (daylight white). Since this is a display application, the basis for color mixing calculations is the set of $1964,10^{\circ} \mathrm{CIE}$ color matching functions [9]. The CIE recommends the use of the colormatching functions of the CIE 1964 Supplementary Stan- dard Colorimetric Observer "whenever correlation with visual color matching of fields of angular subtense greater than about $4^{\circ}$ at the eye of the observer is desired". It has also been noted in the literature that in the optimization of the efficacy of a 3 -wavelength projector there is a trade-off with the color gamut [2]. For this reason, in this study we compare the efficacy with the color gamut area as presented on the CIE 1976 UCS (uniform chromaticity scale) diagram.

The tristimulus values (X, Y and $\mathrm{Z})$ are determined from the color matching functions, $\bar{x}(\lambda), \bar{y}(\lambda)$, and $\bar{z}(\lambda)$, of a given illuminant spectral power distribution $P(\lambda)$ as follows:

$$
G_{n}=\int_{0}^{\infty} P(\lambda) \bar{g}_{n}(\lambda) d \lambda,
$$

where $G_{n}$ and $\bar{g}_{n}(\lambda)$ represent the sequences $(X, Y, Z)$ and $(\bar{x}(\lambda), \bar{y}(\lambda), \bar{z}(\lambda))$ respectively. For the case of a three wavelength combination if we define a matrix $\mathrm{M}$ as

$$
\mathbf{M}=\left[\begin{array}{lll}
\bar{x}\left(\lambda_{r}\right) & \bar{x}\left(\lambda_{g}\right) & \bar{x}\left(\lambda_{b}\right) \\
\bar{y}\left(\lambda_{r}\right) & \bar{y}\left(\lambda_{g}\right) & \bar{y}\left(\lambda_{b}\right) \\
\bar{z}\left(\lambda_{r}\right) & \bar{z}\left(\lambda_{g}\right) & \bar{z}\left(\lambda_{b}\right)
\end{array}\right],
$$

the power of red, green and blue lasers can be calculated by

$$
\mathbf{P}_{r g b}(\boldsymbol{\lambda})=\mathbf{M}^{-1} \mathbf{W},
$$

where the vector $\mathbf{W}$ contains the $x, y$ and $z$ chromaticity coordinates of white D65 point, and $\boldsymbol{\lambda}$ the wavelengths $\lambda_{r}, \lambda_{g}$, and $\lambda_{b}$. In the framework of the CIE $196410^{\circ}$ color-matching functions, the coordinates $\mathrm{x}, \mathrm{y}$ and $\mathrm{z}$ are $0.31382,0.331$ and 0.35518 respectively. $\mathbf{P}_{r g b}$ describes the unique solution of the combination of relative powers of the red, green and blue wavelengths to produce D65 white. For a four-wavelength combination there is no single solution. The combination of four colors is dependent on the relative power of the fourth wavelength. As will be demonstrated this fourth wavelength is consistently found to be in the yellow region and so is represented 
as $\lambda_{y}$. To calculate the four color combination we first define a new vector $\tilde{\mathbf{W}}$ :

$$
\tilde{\mathbf{W}}=\left[\begin{array}{c}
x_{w}-P_{y} \bar{x}\left(\lambda_{y}\right) \\
y_{w}-P_{y} \bar{y}\left(\lambda_{y}\right) \\
z_{w}-P_{y} \bar{z}\left(\lambda_{y}\right)
\end{array}\right]
$$

Expression 3 can be now rewritten in terms of $\tilde{\mathbf{W}}$ for the case of a four wavelength combination as follows:

$$
\mathbf{P}_{r g b}\left(\boldsymbol{\lambda}, \lambda_{y}, P_{y}\right)=\mathbf{M}^{-1} \tilde{\mathbf{W}}
$$

In the former expression, $\mathbf{P}_{r g b}$ depends on $P_{y}$ and $\lambda_{y}$ as well. If we redefine $\boldsymbol{\lambda}$ as the four-dimensional vector $\left[\lambda_{r}\right.$, $\left.\lambda_{g}, \lambda_{b}, \lambda_{y}\right]$, the power vector containing the contribution of all laser sources might be written as follows:

$$
\mathbf{P}=\left[P_{r}\left(\boldsymbol{\lambda}, P_{y}\right), P_{g}\left(\boldsymbol{\lambda}, P_{y}\right), P_{b}\left(\boldsymbol{\lambda}, P_{y}\right), P_{y}\right]
$$

Since the combination of colors is in terms of relative powers we arbitrarily choose to define the powers of each wavelength with respect to the blue wavelength. Hence, we define a new power vector $\tilde{\mathbf{P}}$ as $\mathbf{P} / P_{b}$. Thus, if values for the components red, green, blue and yellow of the vector $\boldsymbol{\lambda}$ and the ratio $P_{y} / P_{b}$ are selected, expression 5 provides a unique result for the ratios $P_{r} / P_{b}$ and $P_{g} / P_{b}$. The total power normalized by $P_{b}$ is

$$
\tilde{\mathrm{P}}_{t}=\frac{P_{r}}{P_{b}}+\frac{P_{g}}{P_{b}}+1+\frac{P_{y}}{P_{b}}
$$

In the same way, using the values $P_{r} / P_{b}, P_{g} / P_{b}$ and $P_{y} / P_{b}$, a normalized flux $\tilde{\mathrm{L}}_{t}$ can be obtained. So, the efficacy is

$$
E_{f f}=\frac{\tilde{\mathrm{L}}_{t}}{\tilde{\mathrm{P}}_{t}}=\frac{L_{t}}{P_{t}}
$$

This method allows us to calculate the efficacy by choosing the relative power of the yellow source with respect to the blue source.

Since the CIE 1976 chromaticity diagram is known to be perceptively relatively linear [10] it is appropriate to quantify the extent of the color gamut as the enclosed area as represented on this plot [5]. In doing so any trade-off between efficacy and gamut can be evaluated. To do this, we firstly have to calculate the $\mathrm{x}, \mathrm{y}$ and $\mathrm{z}$ coordinates for each laser wavelength. These coordinates can be obtained from the following expression:

$$
g_{n}=\frac{G_{n}}{\sum_{n=1}^{3} G_{n}}
$$

where $g_{n}$ represent one element of the sequence $(x, y, z)$. From this set of coordinates, the $u^{\prime}$ and $v^{\prime}$ of the CIE 1976 chart are obtained as follows:

$$
\begin{aligned}
& u^{\prime}=\frac{4 x}{3+12 y-2 x} \\
& v^{\prime}=\frac{9 y}{3+12 y-2 x}
\end{aligned}
$$
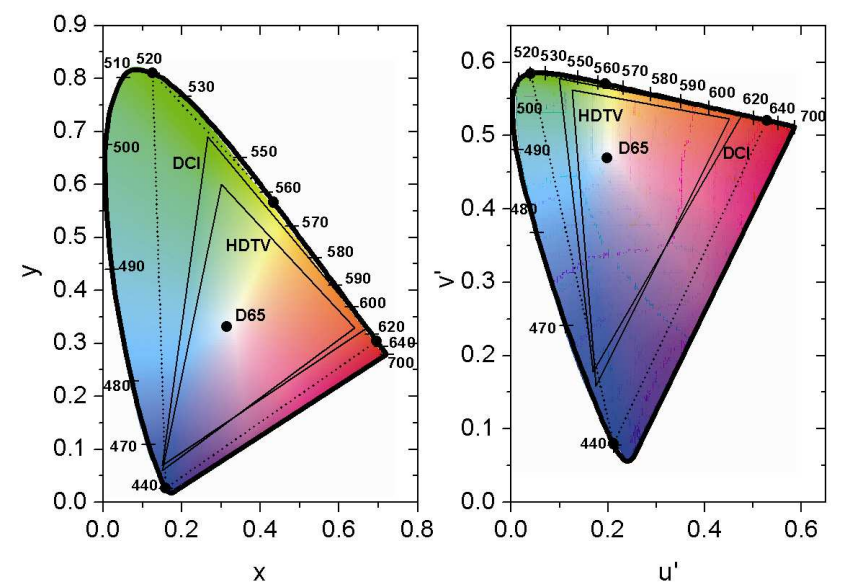

FIG. 1: Left part: CIE 1931 chart. Right part: CIE 1976 chart. In both graphs, achievable color gamuts by the DCI and HDTV input signals have been plotted. Dashed line corresponds to the optimal solution found here using the laser wavelengths $630 \mathrm{~nm}, 520 \mathrm{~nm}, 441 \mathrm{~nm}$ and $563 \mathrm{~nm}$

The color gamut area can be considered as a triangle formed by the coordinates $\left(u_{r}^{\prime}, v_{r}^{\prime}\right),\left(u_{g}^{\prime}, v_{g}^{\prime}\right)$ and $\left(u_{b}^{\prime}, v_{b}^{\prime}\right)$ of the red, green and blue lasers respectively if $\lambda_{g} \geq 520 \mathrm{~nm}$. As fig. 1] shows, the yellow coordinates are in this case almost on the line formed by the green and red coordinates and therefore the contribution of the yellow laser to the formation of a more complex area can be neglected for more simplicity. Thus, under this approximation the color gamut area $(\mathrm{A})$ is:

$$
A=\frac{1}{2}\left[u_{b}^{\prime}\left(v_{r}^{\prime}-v_{g}^{\prime}\right)+u_{g}^{\prime}\left(v_{b}^{\prime}-v_{r}^{\prime}\right)+u_{r}^{\prime}\left(v_{g}^{\prime}-v_{b}^{\prime}\right)\right]
$$

In order to obtain solutions that could be of practical interest and that can be compared with current display devices, we have selected wavelength ranges that try to cover the most important signal inputs, i.e. DCI and HDTV signal inputs plotted in fig. 11 [11, 12]. In this way, a laser projector could display the color spectrum managed by these inputs. The selected wavelength ranges are as follows: $600 \mathrm{~nm} \leq \lambda_{r} \leq 630 \mathrm{~nm}$, $520 \mathrm{~nm} \leq \lambda_{g} \leq 550 \mathrm{~nm}, 440 \mathrm{~nm} \leq \lambda_{b} \leq 470 \mathrm{~nm}$ and $560 \mathrm{~nm} \leq \lambda_{y} \leq 590 \mathrm{~nm}$.

Fig. 2 contains the positive solutions of over 21 million calculations of equation (5) at the above specified wavelength ranges and several power ratios $P_{y} / P_{b}$. The 


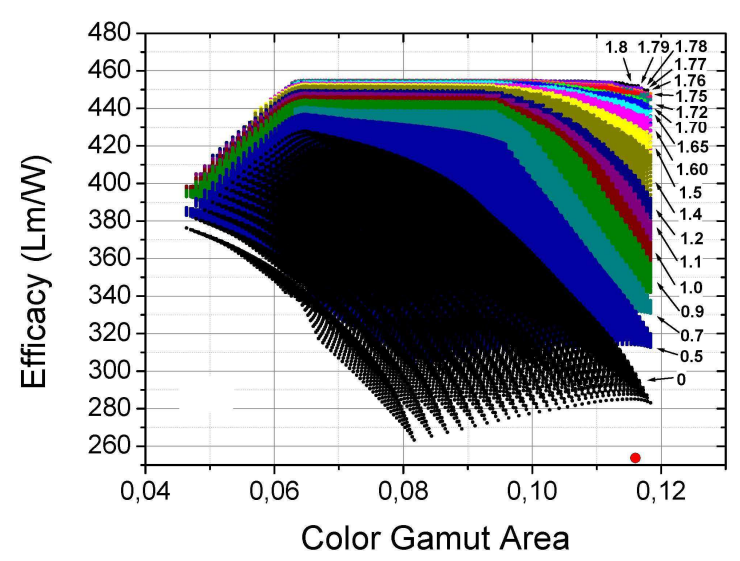

FIG. 2: Efficacy of the white point D65 as function of the color gamut area for different ratios of the yellow laser power to the blue laser power. The big isolated circle denotes the commercial projector described in the text.

efficacy-gamut trade-off of a three color mix is clear. As the power of the fourth wavelength is increased the efficacy of the white point D65 increases at the wavelengths studied. If we choose a figure of merit of the product of efficacy and color gamut area, the most optimum value occurs at $P_{y} / P_{b}=1.8, P_{r} / P_{b}=9 \times 10^{-3}$ and $P_{g} / P_{b}=7 \times 10^{-3}$ and at the wavelengths $630 \mathrm{~nm}$ of the red laser, $520 \mathrm{~nm}$ of the green laser, $441 \mathrm{~nm}$ of the blue laser and $563 \mathrm{~nm}$ of the yellow laser which produce an efficacy of $449.7 \mathrm{~lm} / W$. Since the power needed for the formation of D65 white with the red and green laser is negligible, the calculation results indicate that this choice for both optimum efficacy and color gamut area is mainly obtained with the contributions of the blue and yellow laser. In the $u^{\prime}, v^{\prime}$ space this means that the chromaticity coordinate of the D65 white point is on the straight line formed by the blue laser and yellow laser coordinates. Note that the red and green wavelengths can be considered in the developed method as free variables as well and therefore are obtained as the best optimum wavelengths which together with the blue wavelength maximize the color gamut area in expression 12

By comparison, a commercially available three-color picoprojector (Microvision Show WX [13]) with wavelengths of $442 \mathrm{~nm}, 532 \mathrm{~nm}$ and $642 \mathrm{~nm}$ has an efficacy of $254 \mathrm{~lm} / W$. The performance of this device has been plotted in fig. 2 by means of the coordinates of the efficacy and color gamut area. An addition of a yellow laser at $564 \mathrm{~nm}(\mathrm{Py}=1.76 \mathrm{~Pb})$ would increase the efficacy by $77 \%$ to $451 \mathrm{~lm} / W$.

One additional point is worth mentioning. Fig. 2 shows that there is a very distinct upper limit to the efficacy of any color mix. This occurs at $\sim 455 \mathrm{~lm} / W$. Insofar as the photopic curve presents the maximum efficacy for a single wavelength $(683 \mathrm{~lm} / \mathrm{W}$ at $555 \mathrm{~nm}), 455 \mathrm{~lm} / \mathrm{W}$ has to be understood to be the maximum efficacy with which the color white (D65 point) can be projected by any combination of illumination sources.

The shape of the areas shown in fig. 2 depends on the wavelength ranges used. In fact, if we decrease the color gamut area by using other wavelength ranges closer to the D65 white point in the $u^{\prime}, v^{\prime}$ space, the efficacy would increase up to the maximum value of $\sim 455 \mathrm{~lm} / W$ but at the cost of the color gamut area limiting the capability for obtaining different colors.

In conclusion, we have derived a formulation that permits to calculate the best combination of four wavelengths and powers for laser projection maximizing both color gamut area and efficacy of the D65 white point. The results show that the maximal efficacy projected using the most common video input signals can be only achieved by adding a fourth laser wavelength. Combination of three laser wavelengths would produce less efficacy at suitable color gamuts for projection. The best selection of wavelengths and powers in this framework is found at the wavelengths $630 \mathrm{~nm}, 520 \mathrm{~nm}, 441 \mathrm{~nm}$ and $563 \mathrm{~nm}$ for the red, green, blue and yellow lasers and the power ratio $P_{y} / P_{b}=1.8$ that would produce an efficacy of $449.7 \mathrm{~lm} / W$ when forming a D65 white. These conclusions and results represent a guideline for the future development of picoprojectors and cinema projectors in which particular emphasis is placed on the use of high laser power to increase luminous flux and its implications in safety regulations.

[1] K. V. Chellappan, E. Erden, and H. Urey, "Laser-based displays: a review" Appl. Opt. 49, F79 (2010)

[2] I. Wallhead, R. Ocaña and P. Quinzá, "Designing a laser scanning projector. Part 2: laser safety related issues", Appl. Opt. Vol. 51, 5619 (2012)

[3] E. Buckley, "Eye-safety analysis of current laser-based scanned-beam projection systems," J. Soc. Inf. Disp., 18, 944 (2010).

[4] I. Wallhead, R. Ocaña and P. Quinzá, "Designing a laser scanning picoprojector. Part 1: characteristics of the optical displaying system and color-management-related issues", Appl. Opt. Vol. 51, 4803 (2012)

[5] E. Buckley, "Laser wavelength choices for pico-projector applications", J. Disp. Technol. 7, pp. 402, (2011).

[6] Optimal colors for a laser pico-beamer, Willem Hoving, US Patent Application Publication, US2009/0003390 A1, Jan 1, 2009.

[7] C.H. Brown Elliott, T.L. Credelle, and M.F. Higgins, "Adding a white subpixel," J. Soc. Inf. Disp. 26, (2005).

[8] http://www .aquos-world.com/usen/product/4_color_innovation.

[9] Publication CIE No. 15.2 (1986), Colorimetry, Second edition, Commission Internationale de l'Eclairage (CIE), Central Bureau of the CIE, Vienna, Austria. ISBN 3900 734003

[10] Colorimetry: Understanding the CIE System, Jnos Schanda (Editor), Wiley, 2007 
[11] http://www.itu.int/rec/R-REC-BT.709-5-200204-I/e

[12] SMPTE ST 431-1:2006 D-Cinema Quality - Screen Luminance Level, Chromaticity and Uniformity.
[13] http://www.microvision.com/showwx/pdfs/showwx_userguide.pdf 